\title{
Randomization Validation
}

National Cancer Institute

\section{Source}

National Cancer Institute. Randomization Validation. NCI Thesaurus. Code C115621.

Confirmation that the correct randomization number and treatment assignment have been generated by the randomization program being used in a clinical trial. 